Ein Jahr AMNOG

\title{
GKV feiert, vfa beklagt Strafmodell
}

\section{Die Arzneihersteller ziehen nach einem Jahr Erstattungsbeträge Resümé: Ein faires Verfahren sieht anders aus, kritisiert der vfa. Der GKV-Spitzenverband feiert das Verfahren unterdessen als Erfolg.}

D er Verband forschender Arzneimittelhersteller (vfa) hat das Preisfindungsverfahren, wie es im Arzneimittelmarkt-Neuordnungsgesetz (AMNOG) vorgesehen ist, scharf kritisiert. „Das Verfahren bestraft Innovationen", so vfa-Hauptgeschäftsführerin Birgit Fischer am 30.05.2013 in Berlin. Entgegen der Intention des Gesetzgebers werde das AMNOG als reines Kostendämpfungsinstrument benutzt. „Der Preis neuer, innovativer Arzneimittel soll sich an Preisen von Generika orientieren, die schon lange auf dem Markt sind“, so Fischer. Besonders kritisierte sie die Doppelrolle des GKV-Spitzenverbandes. Dieser ist im Gemeinsamen Bundesausschuss (GBA) bei der Nutzenbewertung beteiligt und Verhandlungspartner bei den Erstattungsbeiträgen. Er nutze diese Macht aus, um bei der Nutzenbewertung eine billige Vergleichstherapie zu wählen und dann die Erstattungsbeträge eines innovativen Arzneimittels mit bestätigtem Zusatznutzen von dem Preis dieser Vergleichstherapie abzuleiten.

Vor gut einem Jahr wurde der erste Erstattungsbetrag nach den Regelungen des neuen AMNOG zwischen einem Pharmaunternehmen und dem GKVSpitzenverband vereinbart.

\section{Zwei Milliarden Euro Einsparziel}

Seither kamen 20 Erstattungsbeträge hinzu. Derzeit laufen zehn Verhandlungen. 17 Erstattungsbeträge wurden zwischen dem jeweiligen Pharmaunternehmen und dem GKV-Spitzenverband ver- einbart. In vier Verhandlungen konnte keine einvernehmliche Lösung gefunden werden und die gesetzliche vorgesehene Schiedsstelle traf dann eine Entscheidung. Zwei weitere Medikamente wurden wegen eines fehlenden Zusatznutzens vom GBA direkt in Festbetragsgruppen eingeordnet. Das Fazit des GKV-Spitzenverbandes ist nach einem Jahr Verhandlungen positiv. „Mit diesem Gesetz wird bei den neuen Medikamenten die Spreu vom Weizen getrennt", so Johann-Magnus von Stackelberg, stellvertretender Vorstandsvorsitzender des GKV-Spitzenverbandes. Durch die 21 Erstattungsbeträge könnten in den Jahren 2012 und 2013 etwa 120 Mio. Euro Arzneimittelausgaben eingespart werden, prognostizierte von Stackelberg - 16\% des Umsatzes dieser Arzneimittel. Die schwarz-gelbe Koalition wollte ursprünglich bis zu zwei Milliarden Euro Arzneimittelkosten pro Jahr mit der frühen Nutzenbewertung sparen. Diese Einsparziele seien nicht unrealistisch, sagte von Stackelberg.

Sie könnten jedoch nur mit der Bewertung des Bestandsmarktes realisiert werden. Nach Angaben des GBA soll die systemische Bewertung des Bestandsmarktes Mitte Juli starten. Sunna Gieseke

\section{AMNOG - insgesamt zwölf onkologische Substanzen bewertet \\ (HL) Von den insgesamt 38 neuen Wirkstoffen, die seit Anfang 2011 auf dem deutschen Markt eingeführt wurden, haben zwölf eine Indikation in der Hämatologie/Onkologie:}

Substanz

Abirateronacetat (Zytiga ${ }^{\oplus}$ )

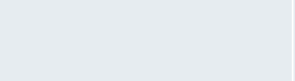

Axitinib (Inlyta $\left.{ }^{\oplus}\right)$

Brentuximabvedotin (Adcetris ${ }^{\circledast}$ )

Cabazitaxel (Jevtana ${ }^{\oplus}$ )

Crizotinib (Xalkori ${ }^{\oplus}$ )

Decitabin (Dacogen $\left.{ }^{\circledast}\right)$

Eribulin (Halaven ${ }^{\oplus}$ )

Ipilimumab $\left(\right.$ Yervoy $\left.^{\circledR}\right)$

Pixantron (Piuvri ${ }^{\odot}$ )

Tegafur/Gimeracil/ Oteracil (Teysuno ${ }^{\oplus}$ ) Vandetanib (Caprelsa $\left.{ }^{\oplus}\right)$

Vemurafenib (Zelboraf ${ }^{\oplus}$ )
Indikation

Subgruppe von Patienten mit metastasiertem kastrationsresistentem Prostatakarzinom mit Progress während oder nach einer Docetaxel-haltigen Chemotherapie, für die eine erneute Behandlung mit Docetaxel nicht mehr in Frage kommen

Patienten mit fortgeschrittenem Nierenzellkarzinom

rezidiviertes oder refraktäres $\mathrm{CD} 30+$ Hodgkin-Lymphom

hormonrefraktäres metastasiertes Prostatakarzinom

Patienten mit vorbehandeltem Anaplastische-Lymphom-Kinase-positivem, fortgeschrittenem, nicht kleinzelligem Lungenkarzinom

akute, myeloische Leukämie lokal fortgeschrittener oder metastasierter Brustkrebs

fortgeschrittenes Melanom

aggressives Non-Hodgkin-B-Zell-Lymphom fortgeschrittener Magenkrebs

aggressives und symptomatisches medulläres Schilddrüsenkarzinom

BRAF-V600 Mutation-positives nicht rezesierbares oder metastasierendes Melanom

\section{Nutzenbewertung}

im Vergleich zu "Best Supportive Care" Hinweis auf einen beträchtlichen

Zusatznutzen

nach vorheriger SunitinibTherapie im Vergleich zu Everolimus kein Zusatznutzen; nach vorheriger Zytokin-Therapie im Vergleich zu Sorafenib Hinweis für einen geringen Zusatznutzen

Zusatznutzen nicht quantifizierbar Hinweis auf geringen Zusatznutzen im Vergleich zu "Best Supportive Care". Anhaltspunkt für einen beträchtlichen Zusatznutzen für ein Subpopulation

geringer Zusatznutzen Anhaltspunkt für geringen Zusatznutzen

Hinweis auf einen beträchtlichen Zusatznutzen

kein Zusatznutzen belegt Zusatznutzen nicht belegt

Zusatznutzen nicht belegt Hinweis auf einen beträchtlichen Zusatznutzen 